Canadian

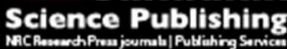

Applied Physiology, Nutrition, and Metabolism Physiologie appliquée, nutrition et métabolisme

\title{
The effects of passive stretching plus vibration on strength and activation of the plantar flexors
}

\begin{tabular}{|r|l|}
\hline Journal: & Applied Physiology, Nutrition, and Metabolism \\
\hline Manuscript ID & apnm-2016-0010.R2 \\
\hline Manuscript Type: & Article \\
\hline Date Submitted by the Author: & 04-Apr-2016 \\
\hline Complete List of Authors: & $\begin{array}{l}\text { Miller, Jonathan; University of Kansas, Health, Sport \& Exercise Science } \\
\text { Herda, Trent; University of Kansas, Health, Sport \& Exercise Science } \\
\text { Trevino, Michael; University of Kansas, Dept. of Health, Sport, and Exercise } \\
\text { Sciences } \\
\text { Mosier, Eric; University of Kansas, Health, Sport \& Exercise Science }\end{array}$ \\
\hline Keyword: & $\begin{array}{l}\text { plantar flexors, vibration, passive stretching, interpolated twitch, passive } \\
\text { range of motion }\end{array}$ \\
\hline &
\end{tabular}

SCHOLARONE $^{\text {Im }}$

Manuscripts 
Title: The effects of passive stretching plus vibration on strength and activation of the plantar flexors

Short Title: Passive stretching and muscle strength

Jonathan D. Miller ${ }^{1}$, Trent J. Herda ${ }^{1}$, Michael A. Trevino ${ }^{1}$, and Eric M. Mosier ${ }^{1}$

${ }^{1}$ Neuromechanics Laboratory, Department of Health, Sport and Exercise Sciences, University of

Kansas, Lawrence, KS 66044

Jonathan D. Miller, j980m842@ku.edu

Trent J.Herda, t.herda@ku.edu

Michael A.Trevino, mtrevino@ku.edu

Eric M. Mosier, emosier4@ku.edu

Author Address: $\quad$ Trent J. Herda, Ph.D.

Assistant Professor - Director, Neuromechanics Laboratory

Dept. Health, Sport, and Exercise Sciences

University of Kansas

1301 Sunnyside Ave, Room 101BE

Lawrence, KS 66045

Office Phone: (785) 864-2224

Fax: (785) 864-3343

Email: t.herda@ku.edu 


\begin{abstract}
This study examined the effects of passive stretching only, (PS+CON) and passive stretching with the addition of continuous vibration during post-passive stretching tests, (PS+VIB) on peak torque $(\mathrm{PT})$, percent voluntary inactivation $(\% \mathrm{VI})$, single stimulus twitch torque $\left(\mathrm{TT}_{\mathrm{SINGLE}}\right)$, and doublet stimuli twitch torque $\left(\mathrm{TT}_{\text {DOUBLET }}\right)$ of the plantar flexors at a short $\left(20^{\circ}\right.$ plantar flexion$[\mathrm{PF}])$ and long muscle length $\left(15^{\circ}\right.$ dorsiflexion$\left.[\mathrm{DF}]\right)$. Fourteen healthy men (age $=22 \pm 3$ years) performed isometric maximal voluntary contractions (MVCs) at PF and DF, and passive range of motion (PROM) assessments before and after 8x30 second passive stretches without (PS+CON) or with VIB (PS+VIB) administered continuously throughout post-passive stretching tests. The passive properties of the muscle tendon unit (MTU) were assessed pre- and postpassive stretching via PROM, passive torque (PASSTQ) and musculotendinous stiffness (MTS) measurements. PT, $\mathrm{TT}_{\text {SINGLE }}$, and $\mathrm{TT}_{\text {DOUBLET }}$ decreased, whereas, \%VI increased following passive stretching at $\mathrm{PF}$ and $\mathrm{DF}(\mathrm{P}<0.05)$ with no significant differences between $\mathrm{PS}+\mathrm{CON}$ and PS+VIB. PASSTQ and MTS decreased while PROM increased post-passive stretching during both trials $(\mathrm{P}<0.05)$. The stretching-induced force/torque deficit and increases in \%VI were evident following passive stretching at short and long muscle lengths. Although not statistically significant, effect size calculations suggested large and moderate differences in the absolute changes in PT (Cohen's D = 1.14) and \%VI (Cohen's D = 0.54) from pre- to post-passive stretching between treatments, with PS+VIB having greater decreases PT and higher \%VI than PS + CON. The decrement in PT following passive stretching may be primarily neural in origin.
\end{abstract}

Keywords: plantar flexors, vibration, passive stretching, passive range of motion, interpolated twitch 


\begin{tabular}{l|l} 
acronym & definition \\
\hline PS+CON & treatment: passive stretching with no vibration \\
PS+VIB & treatment: passive stretching with vibration \\
PT & peak torque \\
$\%$ VI & percent voluntary inactivation \\
TT $_{\text {SINGLE }}$ & single stimulus twitch torque \\
TT $_{\text {DOUBLET }}$ & doublet stimuli twitch torque \\
PF & joint angle: $20^{\circ}$ plantar flexion \\
DF & joint angle: $15^{\circ}$ dorsiflexion \\
MVC & maximal voluntary contraction \\
PROM & passive range of motion \\
VIB & vibration \\
MTU & muscle tendon unit \\
PASSTQ & passive torque \\
MTS & musculotendinous stiffness \\
ITT & interpolated twitch technique \\
EMG & electromyography \\
SOL & soleus muscle \\
M-wave & maximal compound muscle action potential \\
RMS & root mean squared
\end{tabular}




\section{INTRODUCTION}

Passive stretching is still widely used as a means of warm-up and injury prevention prior to competitive and recreational sports despite numerous studies reporting acute decreases in force production following bouts of passive stretching (Avela et al. 1999; Behm et al. 2001; Cramer et al. 2004; Cramer et al. 2005; Evetovich et al. 2003; Fowles et al. 2000; Herda et al. 2008; Herda et al. 2010; Herda et al. 2009; Kokkonen et al. 1998; Marek et al. 2005; Nelson et al. 2001a; Nelson et al. 2001b; Orizio et al. 2003; Ryan et al. 2014; Young and Elliott 2001). The loss in force following passive stretching has been well documented; however, the exact mechanism causing the decrease in force remains unclear. Two main hypotheses have been proposed to explain this phenomenon: 1) neural deficits via diminished muscle spindle-mediated Ia afferent activity and/or gamma loop function, and 2) mechanical alterations associated with changes in the optimal force producing conditions along the length-tension relationship (Avela et al. 1999; Behm et al. 2001; Cramer et al. 2004; Evetovich et al. 2003; Fowles et al. 2000; Herda et al. 2008; Herda et al. 2009; Kokkonen et al. 1998; Nelson et al. 2001a; Nelson et al. 2001b; Ryan et al. 2014; Young and Elliott 2001).

Numerous studies have reported decreases in maximal strength accompanied with reduced muscle activation (Behm et al. 2001; Cramer et al. 2005; Fowles et al. 2000; Herda et al. 2008; Herda et al. 2009; Ryan et al. 2014). For example, Herda et al. (2008) reported a decrease in isometric force and muscle activation following 20 minutes of passive stretching of the plantar flexors. The authors also reported similar reductions in isometric force and muscle activation following 20 minutes of a vibration only treatment and, therefore, the authors proposed that prolonged stretching diminishes muscle spindle and/or gamma loop function in a similar manner to vibration. Muscle spindle function is necessary to facilitate and recruit all available motor units during a maximal voluntary contraction; therefore, muscle spindle dysfunction would 
reduce overall muscle strength via incomplete activation of the motoneuron pool (Kouzaki et al. 2000; Ryan et al. 2014). It is plausible that the continued mechanical lengthening of muscle spindles during passive stretching may reduce Ia afferent activity and diminish maximal isometric force.

In addition to diminished neural function, previous studies have reported muscle length dependent changes in maximal isometric force following passive stretching (Balle et al. 2015; Herda et al. 2008; McHugh and Johnson 2006). Herda et al. (2008) reported the stretchinginduced force deficit following passive stretching at short muscle lengths, however, there were no difference at long muscle lengths for the leg flexors. McHugh and Johnson (2006) reported similar findings and suggested that passive stretching may shift the length-tension relationship rightward; thus, there would be decreases in maximal strength at the shorter lengths but possible increases in maximal strength at longer muscle lengths. It remains unclear if this phenomenon occurs for the plantar flexors following passive stretching.

The purpose of this study was to further elucidate the effects of passive stretching on maximal strength of the plantar flexors at a short and long muscle length with the use of continuous vibration to further determine the extent of neural deficits following a moderate duration of passive stretching. Vibration is a valuable tool that has been incorporated in numerous studies to remove muscle spindle support to the central nervous system (Bongiovanni et al. 1990; Herda et al. 2009; Kouzaki et al. 2000; Richardson et al. 2006). In addition, nonvoluntary evoked twitches were performed at rest and during a MVC to better assess possible differences in mechanical mechanisms and voluntary inactivation as a result of passive stretching at short and long muscle lengths. It is hypothesized that the passive stretching only treatment, $(\mathrm{PS}+\mathrm{CON})$ and the treatment in which vibration is applied following passive stretching and is 
continuous throughout the remainder of the experiment (PS+VIB) will behave similarly. This would indicate neural mechanisms account for the majority of the strength loss following passive stretching. Furthermore, if the stretching-induced force deficit is neural in origin, then both muscle lengths (PF and DF) should show similar strength deficits and, thus, would indicate the strength loss is not primarily result of mechanical mechanisms (i.e., length-tension relationship). 


\section{MATERIALS AND METHODS}

\section{Subjects}

Fourteen healthy men (mean $\pm \mathrm{SD}$ age $=22 \pm 3$ years, stature $=177 \pm 6 \mathrm{~cm}$; mass $=84 \pm$ $11 \mathrm{~kg}$ ) participated in this study. None of the participants reported any current or ongoing neuromuscular diseases or musculoskeletal injuries specific to the ankle, knee, or hip joints. This study was approved by the University Institutional Review Boards for Human Subjects, and all participants completed a written informed consent form and a Pre-Exercise Testing Health \& Exercise Status Questionnaire.

Research design

The participants visited the laboratory three times separated by at least 2 days. Subjects were asked to refrain from strenuous physical activity for 24 hours before any testing. The first visit was a familiarization trial followed by two experimental visits. During the familiarization visit participants practiced all assessments and stretching procedures. For each experimental visit, measurements were completed prior (pre) and following (post) $8 \times 30$ seconds of PS. One of the two experimental trials, in random order, included Achilles tendon vibration (PS+VIB) during the post-passive stretching measurements, while the other experimental trial had no VIB post-passive stretching (PS+CON). Pre- and post-passive stretching measurements included passive range of motion (PROM) assessments, evoked twitches at rest and maximal voluntary contractions (MVCs) with superimposed and potentiating twitches administered to calculate percent voluntary inactivation $(\% \mathrm{VI})$. Peak twitch torque was recorded from the single stimuli

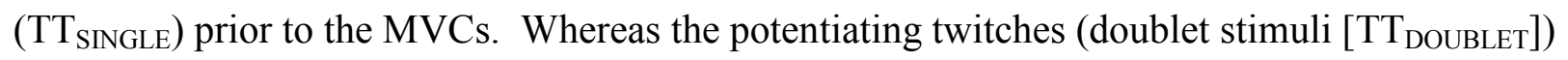
were recorded following the MVCs. The evoked twitches and MVCs were performed at joint angles $+15^{\circ}$ (dorsiflexion $\left.[\mathrm{DF}]\right)$ and $-20^{\circ}$ (plantar flexion $[\mathrm{PF}]$ ) relative to neutral $\left(90^{\circ}\right.$ between the foot and leg) in random order (Figure 1). 


\section{Isometric Testing}

Each participant was seated with restraining straps over the right thigh, pelvis, and trunk, and the lateral malleolus of the fibula was aligned with the input axis of the isokinetic dynamometer (Biodex System 3; Biodex Medical Systems, Inc., Shirley New York) in accordance with the Biodex User's guide ( Biodex Pro Manual, applications/Operations, 1998). All isometric plantar flexor strength assessments were performed on the right leg at the DF and PF joint angles with $0^{\circ}$ of flexion in the right knee. To determine voluntary peak torque (PT), evoked twitch peak torques, $\left(\mathrm{TT}_{\mathrm{SINGLE}}\right.$ and $\left.\mathrm{TT}_{\text {DOUBLET }}\right)$ and $\% \mathrm{VI}$, each participant received three evoked single stimuli followed by a 5 second MVCs of the plantar flexors at the DF and PF joint angles. The MVC trials were used to assess PT (before the superimposed twitch) as well as \%VI and $\mathrm{TT}_{\text {DOUBLET }}$ via the interpolated twitch technique (ITT). PT was obtained by averaging the highest 200 data points $(0.10 \mathrm{~s})$ of the digital torque signal prior to the superimposed twitch, where the onset of the superimposed twitch was determined manually by the investigators from the electromyographic (EMG) signal simultaneously recorded from the soleus (SOL) muscle. The highest PT value, with subsequent \%VI, of the highest two pre-passive stretching MVC trials were used as the representative scores for further analyses. Only one MVC was completed at each ankle joint angle following passive stretching. Two minutes of rest were given following the post-passive stretching PROM tests for both treatments. For the PS+VIB treatment, VIB was initiated at the beginning of the 2-minute rest period and was continuous from that point until the end of the experiment, which included VIB being applied during post-passive stretching strength measurements. A relatively short 2-minute rest $(\mathrm{PS}+\mathrm{CON})$ or $\mathrm{VIB}(\mathrm{PS}+\mathrm{VIB})$ period was chosen because that duration has been shown to be sufficient for VIB to attenuate force production 
(Bongiovanni et al. 1990), while still allowing time for remaining tests to be completed before the force attenuating effects of stretching cease (Ryan et al. 2008).

The duration of time (mean $\pm \mathrm{SD}$ ) between the PROM and isometric testing at the first joint angle was $211 \pm 39 \mathrm{sec}$ and $212 \pm 49 \mathrm{sec}$ for the PS+CON and PS+VIB trials, respectively and was $350 \pm 64 \mathrm{sec}$ and $332 \pm 77 \mathrm{sec}$ for the second joint angle for the PS+CON and PS+VIB, respectively.

\section{Evoked Twitches}

Transcutaneous electrical stimuli were delivered to the tibial nerve using a high-voltage constant-current stimulator (Digitimer DS7AH, Herthfordshire, UK). Single stimuli were applied via a bipolar probe with saline-soaked tips in order to determine the optimal probe location and the maximal compound muscle action potential (M-wave) via EMG of the SOL with incremental amperage increases (2-20mA). Once a plateau in the peak-to-peak M-wave was determined, despite amperage increases, $20 \%$ was added to the amperage that yielded the highest peak-topeak M-wave to assure supramaximal stimulus. The bipolar probe was then replaced by two adhesive electrodes (Disposable 4-Disk Electrodes; Technomed Europe; Maastricht Airport, The Netherlands) that were used to administer stimuli for all experimental twitches. A single stimulus was a 1-ms duration square wave impulse, while a doublet consisted of two single stimuli delivered successively at $100 \mathrm{~Hz}$. Three single stimuli twitches were used at rest prior to the MVCs and recorded as $\mathrm{TT}_{\mathrm{SINGLE}}$. $\mathrm{TT}_{\mathrm{SINGLE}}$ was obtained by averaging the highest 10 data points $(0.005 \mathrm{~s})$ of the digital torque signal. The average of the three single stimuli twitches were used for further analyses. Doublets were administered during the MVC trials (superimposed twitch) to increase the signal-to-noise ratio and minimize the series elastic effects on torque production 
(Desbrosses et al. 2006). The potentiated twitch was administered $3-5$ seconds following the MVC to calculate \%VI according to the ITT.

$$
\% V I=100-\left\{\left[1-\left(\frac{\text { superimposed twitch }}{\text { potentiated twitch }}\right)\right] \times 100\right\}
$$

The three resting twitches and MVCs with the superimposed and potentiated twitches were performed at both joint angles before and 2 minutes after the PROM assessment. The peak twitch torque from the potentiated twitch was recorded as the $\mathrm{TT}_{\text {DOUBLET }}$.

\section{Passive Range of Motion}

The PROM of the plantar flexors was determined for each participant immediately preceding and immediately following PS. PROM was performed using the isokinetic dynamometer programmed in passive mode. The dynamometer lever arm passively dorsiflexed the foot at an angular velocity of $5 \%$ s until the point of discomfort, but not pain, as verbally acknowledged by the subject. PROM was calculated as the range of motion attained from a neutral joint angle to the maximum tolerable point of passive dorsiflexion as measured with the position signal from the dynamometer. No gravity correction was performed based on the methods of (Muir et al. 1999) who indicated that the foot constituted approximately $1.4 \%$ of the body's mass (Winter 1990) and suggested that this mass can be considered negligible.

\section{Musculotendinous Stiffness}

MTS of the plantar flexors was quantified using a fourth-order polynomial regression model that was fitted to the passive angle-torque curves for each subject according to the procedure described by (Nordez et al. 2006). MTS quantifies the joint angle-specific stiffness of the muscle tendon unit (MTU) based on the passive angle-torque relationship. MTS was calculated for each degree during the passive angle-torque relationships from the neutral ankle position (i.e., 90 between the foot and leg) to the end of the range of motion. Only MTS values at 
the DF joint angle $\left(+15^{\circ}\right.$ dorsiflextion $)$ for each trial were used for analysis. MTS values were calculated with the following equation (Nordez et al. 2006), where $\theta$ represented the joint angle, and $m, n, o, p$, and $q$ were coefficients in the fourth-order polynomial regression model that was fit to the passive angle-torque relationship:

$$
\text { passive torque }=m \theta^{4}+n \theta^{3}+o \theta^{2}+p \theta+q
$$

MTS was subsequently calculated with the following equation (Nordez et al., 2006):

$$
\mathrm{MTS}=4 m \theta^{3}+3 n \theta^{2}+2 o \theta+p
$$

\section{Surface Electromyography}

A bipolar surface EMG electrode (Delsys, Inc., Boston, MA) was placed on the SOL muscle. The electrode was placed along the longitudinal axis of the tibia at $67 \%$ of the distance between the medial condyle of the femur and the medial malleolus. The reference electrode (Dermatrode HE-W, American Imex; Irvine, California, USA) was placed on the contralateral patella. Before sensor placement, the surface of the skin was prepared by shaving, removing superficial dead skin with adhesive tape, and sterilizing with an alcohol swab. To remove the dead layers of skin, hypoallergenic tape (3M, St. Paul, Minnesota) was applied to the site, then peeled back to remove contaminants (Delsys dEMG User Guide). This process was repeated multiple times.

\section{Signal Processing}

The EMG, position and torque signals were recorded simultaneously with a National Instruments data acquisition system (cDAQ-9174, National Instruments; Austin, Texas, USA) during the evoked twitches, isometric MVCs, and PROMs. The torque $(\mathrm{Nm})$ and position $\left(^{\circ}\right)$ signals from the dynamometer and the EMG $(\mathrm{mV})$ signal recorded from the SOL were sampled at $2 \mathrm{kHz}$. All signals were stored on a personal computer (Dell Optiplex 9010, Dell, Inc., Round 
Rock, Texas, USA) and processing was completed off-line using custom written software (LabVIEW v 12.0, National Instruments, Austin, Texas, USA). The EMG signals were digitally filtered (zero-phase fourth - order Butterworth) with a pass band of $10-500 \mathrm{~Hz}$, whereas, the torque and position signals where low-pass filtered with a $10 \mathrm{~Hz}$ cutoff (zerophase fourth - order Butterworth) and gravity corrected so that the baseline torque value was $0 \mathrm{Nm}$. All subsequent analyses were performed on the filtered signals. The time domain of the EMG was represented as the root mean square (RMS) amplitude value. EMGRMS was collected during stretches to ensure the movements were passive in accordance to Gajdosik et al. (2005).

\section{Passive Stretching}

The repeated passive stretching of the right plantar flexor muscles were performed on the isokinetic dynamometer in the same fashion as the PROM assessments. The dynamometer passively dorsiflexed the foot until the pre-determined torque threshold was met. The predetermined torque threshold was obtained for each individual during the during the familiarization visit as the point of discomfort, but not pain, as verbally acknowledged by the subject. The dynamometer maintained this constant passive torque which stretched the plantar flexors 8 times lasting $30 \mathrm{~s}$ each. This tension was released for a minimal rest period $(5 \mathrm{~s})$ between each stretch.

\section{Vibration}

A continuous VIB was applied to the Achilles tendon using a Techno Concept Vibrasense VB 200 massager (Pitaugier Mane, France). The VIB frequency was set at $55 \mathrm{~Hz}$ and started immediately following the post-passive stretching PROM tests. VIB was administered for 2 minutes prior to the start of the post-passive stretching isometric muscle actions and was continuous during the remainder of testing, which included VIB being applied during the post- 
passive stretching isometric muscle actions. Previous research has demonstrated that two minutes of VIB decreases maximal strength (Bongiovanni et al. 1990). In addition, pilot data confirmed that a 2-minute continuous vibration protocol was sufficient to decrease MVC force. Therefore, 2-minutes of VIB would reduce MVC strength while the stretching induced force deficit remained intact. During the PS + CON trial (no VIB), the vibrator was strapped to the Achilles tendon but remained off.

Statistical analysis

Four separate three-way repeated measures ANOVAs [time (pre- vs post-passive stretching $) \times$ treatment $(\mathrm{PS}+\mathrm{CON}$ vs $\mathrm{PS}+\mathrm{VIB}) \times$ angle $(\mathrm{DF}$ vs $\mathrm{PF})]$ were used to analyze the PT, $\mathrm{TT}_{\text {SINGLE}}, \% \mathrm{VI}, \mathrm{TT}_{\text {DOUBLET. }}$ Three separate two-way repeated measures ANOVAs [time (pre- vs post-passive stretching) $\times$ treatment $(\mathrm{PS}+\mathrm{CON}$ vs $\mathrm{PS}+\mathrm{VIB})]$ were used to analyze MTS and passive torque (PASSTQ) at the DF angle, and PROM. When appropriate, follow-up analyses were performed using dependent samples t-tests with Bonferroni's corrections. An $\alpha$ of 0.05 was used to determine statistical significance. Statistical analyses was performed using SPSS v. 12.0 (SPSS Inc., Chicago, Illinois, USA). 


\section{RESULTS}

Mean $\pm \mathrm{SD}$ values for the strength $\left(\mathrm{PT}, \mathrm{TT}_{\mathrm{SINGLE}}, \mathrm{TT}_{\mathrm{DOUBLET}}\right.$, and \%VI) and PROM (PROM, PASSTQ, and MTS) data for the pre- and post-passive stretching trials are presented in tables 1 and 2, respectively.

Passive Range of Motion

There was no significant two-way interaction (time $\times$ treatment, $\mathrm{P}=0.390$ ) and no significant main effect for treatment $(\mathrm{P}=0.353)$. However, there was a significant main effect for time $(\mathrm{P}=0.006)$. PROM increased from pre- to post-passive stretching (mean $\pm \mathrm{SD}, 9.9 \pm$ 13.0\%) collapsed across PS+CON and PS+VIB.

Passive Torque

There was no significant two-way interaction (time $\times$ treatment, $\mathrm{P}=0.980$ ) and no significant main effect for treatment $(\mathrm{P}=0.304)$. There was, however, a significant main effect for time $(\mathrm{P}=0.002)$. PASSTQ decreased at the DF angle from pre- to post-passive stretching ($13.5 \pm 8.0 \%$ ) collapsed across PS+CON and PS+VIB.

\section{Musculotendinous stiffness}

There was no significant two-way interaction (time $\times$ treatment, $\mathrm{P}=0.295$ ) and no significant main effect for treatment $(\mathrm{P}=0.429)$. There was a significant main effect for time $(\mathrm{P}$ $=0.033)$. MTS decreased at the DF angle from pre- to post-passive stretching $(-18.7 \pm 16.1 \%)$ collapsed across the PS+CON and PS+VIB.

\section{Peak Torque}

There was no significant three-way interaction (time $\times$ treatment $\times$ angle, $\mathrm{P}=0.468$ ), no significant two-way interactions for treatment $\times$ angle $(\mathrm{P}=0.899)$ and angle $\times$ time $(\mathrm{P}=0.056)$, but there was a significant two-way interaction for time $\times$ treatment $(\mathrm{P}=0.017)$. After 
collapsing across angle, dependent-samples t-tests indicated a significant decrease in PT following $\mathrm{PS}+\mathrm{CON}(\mathrm{P}<0.001,-8.6 \pm 10.1 \%)$ and $\mathrm{PS}+\mathrm{VIB}(\mathrm{P}<0.001,-19.1 \pm 8.6 \%)$ (Figure 2A). Although no statistical difference was found between PS $+\mathrm{CON}$ and PS+VIB for the pre- (P $=0.660)$ and post-passive stretching $(\mathrm{P}=0.132)$, the effect size of the difference between treatments post-passive stretching was large (Cohen's $\mathrm{D}=1.14)$. In addition, there was a main effect for angle $(\mathrm{P}<0.001)$. PT was greater at DF than PF collapsed across time and treatment. Doublet Twitch Torque

There was no significant three-way interaction (time $\times$ treatment $\times$ angle, $\mathrm{P}=0.112$ ), no significant two-way interactions for treatment $\times$ angle $(\mathrm{P}=0.225)$, angle $\times$ time $(\mathrm{P}=0.630)$, treatment $\times$ time $(P=0.300)$, and no significant main effect for treatment $(P=0.432)$. However, there were significant main effects for angle $(\mathrm{P}<0.001)$ and time $(\mathrm{P}=0.001)$. TT $_{\text {DOUBLET }}$ was greater at DF than PF collapsed across treatment and time and decreased from pre- to postpassive stretching $(-8.4 \pm 8.2 \%)$ collapsed across treatment and angle (Figure 2B). Single Twitch Torque

There was no significant three-way interaction (time $\times$ treatment $\times$ angle, $\mathrm{P}=0.431$ ), no significant two-way interactions for treatment $\times$ angle $(\mathrm{P}=0.070)$, angle $\times$ time $(\mathrm{P}=0.422)$, treatment $\times$ time $(P=0.217)$, and no significant main effect for treatment $(P=0.144)$. However, there were significant main effects for angle $(\mathrm{P}<0.001)$ and time $(\mathrm{P}<0.001)$. TT $\mathrm{SINGLE}$ Was greater at DF than PF collapsed across treatment and time and decreased $(-10.4 \pm 7.4 \%)$ from pre- to post-passive stretching collapsed across treatment and angle (Figure 2C).

\section{Percent Voluntary Inactivation}

There was no significant three-way interaction (time $\times$ treatment $\times$ angle, $\mathrm{P}=0.238$ ), no significant two-way interactions for treatment $\times$ angle $(\mathrm{P}=0.775)$, angle $\times$ time $(\mathrm{P}=0.447)$, and 
treatment $\times$ time $(\mathrm{P}=0.114)$, and no significant main effect for treatment $(\mathrm{P}=0.683)$. However, there were significant main effects for angle $(\mathrm{P}=0.008)$ and time $(\mathrm{P}=0.002) . \% \mathrm{VI}$ increased $(10.3 \pm 10.1 \%)$ from pre- to post-passive stretching collapsed across angle and treatment (Figure 2D). \%VI was greater at DF than PF collapsed across treatment and time (Table 1). Despite no significant differences, there was a medium effect size (Cohen's D $=0.535)$ between PS $+\mathrm{CON}$ $(6.9 \pm 9.7 \% \mathrm{VI})$ and $\mathrm{PS}+\mathrm{VIB}(13.7 \pm 14.8 \% \mathrm{VI})$ treatments for post-passive stretching measurements of \%VI. 


\section{DISCUSSION}

As expected, decreases in MTS and PASSTQ with concurrent increases in PROM occurred following the PS $+\mathrm{CON}$ and PS+VIB treatments. In addition, PT decreased from pre- to post-passive stretching for PS+CON (-8.6\%) and PS+VIB (-19.1\%) (Figure 2A). Although the decrease in PT was not significantly different between treatments, the effect size of 1.14 (Cohen's D) suggested that there was a difference in post-passive stretching between PS+VIB and PS $+\mathrm{CON}$ treatments. This greater decrease in PT for the PS+VIB treatment was accompanied with a greater increase in $\% \mathrm{VI}(\mathrm{PS}+\mathrm{VIB}=13.7 \% \mathrm{VI})$ in comparison to $\mathrm{PS}+\mathrm{CON}$ treatment $(6.9 \% \mathrm{VI})$, which had a moderate effect size of 0.535 . Thus, suggesting that PS+VIB may have diminished muscle spindle function more so than PS $+\mathrm{CON}$ which resulted in a greater decrease in PT. In addition, the treatments caused similar changes in these parameters at both muscle lengths. No rightward shift in the length-tension relationship as a result of passive stretching provided tentative evidence that the stretching-induced force deficit was primarily neural despite decreases in MTS and PASSTQ at the tested muscle lengths.

In the present study, moderate duration passive stretching reduced PT (-8.6\%) despite 2 minutes of rest prior to the post-passive stretching MVCs. Ryan et al. (2008) examined the doseresponse relationship of the stretching-induced force deficit of the plantar flexors and reported just over a $4 \%$ decrease in PT following 4 minutes of passive stretching with PT remaining slightly under $4 \%$ lower than baseline following 10 minutes of rest. Thus, there was a minimal difference in PT between immediately after and 10 minutes post-passive stretching. It should be of note, that the percent difference in Ryan et al. (2008) was not significantly different from the control and baseline at 10 minutes. In the present study, the greater decrease in PT following passive stretching in comparison to Ryan et al. (2008) may be the result of the muscle lengths 
tested. Nonetheless, there was likely only a minimal change in the stretching-induced force deficit from immediately following to post-passive stretching tests. In addition, continuous VIB lasting 10-30 seconds has been reported to diminish muscle spindle function with Bongiovanni et al. (1990) demonstrating that the VIB-induced force deficit was present following 2 minutes of VIB. Therefore, the stretching-induced deficit likely remained intact during the 2 minutes of continuous VIB that was intended to completely diminish muscle spindle function.

It is hypothesized that the primary contributors to the stretching-induced force deficit following prolonged passive stretching are neural mechanisms (Avela et al. 1999; Behm et al. 2001; Ryan et al. 2014). Specifically, it is believed that muscle spindle activation is reduced with a subsequent reduction in Ia afferent activity to the central nervous system following prolonged PS, which results in decreased muscle activation. VIB of $>30$ seconds has been directly shown to diminish muscle spindle activity and subsequent Ia afferent feedback to the central nervous system (de Ruiter et al. 2003; Kouzaki et al. 2000; Pope and DeFreitas 2015; Ushiyama et al. 2005). In theory, the addition of VIB to the post-passive stretching measurements would not further impair force or muscle activation if the primary mechanism of the stretching-induced force deficit was muscle spindle mediated. However, there was a greater decrease in force for the PS+VIB than the PS+CON treatment as indicated by the effect size. There was also a greater increase in \%VI from pre- to post-passive stretching for the PS+VIB treatment. Thus, it is plausible that the addition of VIB caused further inhibition of the muscle spindles, resulting in greater muscle inactivation and further decreased PT.

MTS and PASSTQ data indicated that mechanical changes were observed in the MTU as a result of PS. Thus, mechanical mechanisms cannot be completely discounted as a contributor to the stretching-induced force deficit. In the present study $\mathrm{TT}_{\text {DOUBLET }}(-8.4 \%)$ and $\mathrm{TT}_{\text {SINGLE }}(-$ 
10.4\%) decreased from pre- to post-passive stretching when collapsed across angle and treatment (figure 2B,C). Although not statistically tested, the percent decrease in $\mathrm{TT}_{\text {DOUBLET }}$ was less than the decrease in $\mathrm{TT}_{\text {SINGLE}}$, which suggested the decrease in twitch force due to mechanical changes in the MTU can be partially overcome by a doublet stimulus. Furthermore, Behm et al. (2001) reported that twitch force decreased after 20 minutes of passive stretching but the decrease was not present during a $300 \mathrm{~ms}$ tetanic stimulation at $100 \mathrm{hz}$, which indicated that the continued firing of motor units was sufficient to overcome the increased muscle compliance caused by stretching. Therefore, the loss of force due to mechanical alterations (i.e., decreased MTS) is likely not the primary mechanism of the decreased maximal isometric strength following PS.

Previously, it has been reported that the stretching-induced force deficit is muscle length dependent (Balle et al. 2015; Herda et al. 2008; McHugh and Johnson 2006). For example, McHugh and Johnson (2006), Herda et al. (2008), and Balle et al. (2015) reported significant decreases in PT at shorter muscle lengths with no change in strength at the longer muscles lengths during leg flexion, while Nelson et al. (2001a) reported similar findings for the leg extensors. Therefore, it was hypothesized that passive stretching causes acute alterations in the length-tension relationship, such as, a rightward shift in the relationship. However, this was unfounded as force was reduced at both muscle lengths in a similar manner. The difference in the findings is likely related to the muscle tested and the stretching protocol implemented. Studies that reported no reduction in strength at the longer muscle lengths following stretching were performed on the leg flexors or extensors, whereas, the plantar flexors were tested in the present study. In addition, these previous studies did not incorporate a constant-torque stretching protocol that results in muscle creep as in the present study, but rather used unassisted and 
assisted partner stretches. Nonetheless, the stretching-induced force deficit was found to occur at a shorter and longer muscle length in the plantar flexors, which contradicts the findings from experiments performed on the leg flexors and extensors using unassisted and assisted partner stretches. In addition, similar decreases in strength at the short and long muscle lengths, rather than a rightward shift in the length-tension relationship, may provide further evidence that the stretching-induced force deficit as a result of a moderate dosage of passive stretching of the plantar flexors is primarily neural.

Limitation to our interpretation is that there was no vibration only trial, which does not allow for a direct interpretation of the effects of the 2-minute vibration protocol. However, decreases in strength as a result of short duration continuous vibration (2-minutes) applied during strength testing has been previously reported in Bongiovanni et al. (1990). Furthermore, pilot testing from our laboratory indicated that 2-minutes of vibration would reduce strength when applied during the MVCs. In addition, the ANOVA models indicated that there were no significant differences between the PS $+\mathrm{CON}$ and PS + VIB trials for decreases in strength and muscle activation, however, large and medium effect sizes for these differences were found (Cohen's $\mathrm{D}=1.14$ and 0.535 ). Still, caution is warranted when interpreting the results that vibration further reduced strength and muscle activation. Future studies should confirm the effects of a relatively short, 2-minute continuous vibration protocol on strength and muscle activation.

Effect sizes suggested that there were large and moderate differences, although not statistically significant, in post-passive stretching PT and \%VI between PS+VIB and PS+CON treatments with greater changes occurring for the PS+VIB than PS+CON. PT decreased similarly at both long and short muscle lengths, which is contradictory to previous studies using 
unassisted and assisted partner stretching on the leg flexors and extensors. In conclusion, the stretching-induced force deficit as a result of constant-torque passive stretching appears to be primarily neural in origin at short and long muscle lengths of the plantar flexors. However, VIB reduced muscle spindle function to a greater extent than passive stretching alone. Future research should examine more realistic stretching routines that would translate to exercise- or sportperformance as it is likely that a short duration ( $\sim 30 \mathrm{~s})$ of stretching may not result in a force deficit.

\section{ACKNOWLEDGEMENTS}

The authors would like to thank undergraduate research assistants Lauren Marquess, Omar Rehman, and Jeremy Lippman for their efforts in data collection and analyses.

\section{CONFLICTS OF INTEREST}

The authors declare that there are no conflicts of interest. 


\section{REFERENCES}

Avela, J., Kyrolainen, H. and Komi, P. V. 1999. Altered reflex sensitivity after repeated and prolonged passive muscle stretching. J Appl Physiol (1985). 86: 1283-1291.

Balle, S. S., Magnusson, S. P. and McHugh, M. P. 2015. Effects of contract-relax vs static stretching on stretch-induced strength loss and length-tension relationship. Scand J Med Sci Sports.

Behm, D. G., Button, D. C. and Butt, J. C. 2001. Factors affecting force loss with prolonged stretching. Can J Appl Physiol. 26: 261-272.

Bongiovanni, L. G., Hagbarth, K. E. and Stjernberg, L. 1990. Prolonged muscle vibration reducing motor output in maximal voluntary contractions in man. J Physiol. 423: 15-26.

Cramer, J. T., Housh, T. J., Johnson, G. O., Miller, J. M., Coburn, J. W. and Beck, T. W. 2004. Acute effects of static stretching on peak torque in women. J Strength Cond Res. 18: 236-241.

Cramer, J. T., Housh, T. J., Weir, J. P., Johnson, G. O., Coburn, J. W. and Beck, T. W. 2005. The acute effects of static stretching on peak torque, mean power output, electromyography, and mechanomyography. Eur J Appl Physiol. 93: 530-539.

de Ruiter, C. J., van der Linden, R. M., van der Zijden, M. J., Hollander, A. P. and de Haan, A. 2003. Shortterm effects of whole-body vibration on maximal voluntary isometric knee extensor force and rate of force rise. Eur J Appl Physiol. 88: 472-475.

Desbrosses, K., Babault, N., Scaglioni, G., Meyer, J. P. and Pousson, M. 2006. Neural activation after maximal isometric contractions at different muscle lengths. Med Sci Sports Exerc. 38: 937-944.

Evetovich, T. K., Nauman, N. J., Conley, D. S. and Todd, J. B. 2003. Effect of static stretching of the biceps brachii on torque, electromyography, and mechanomyography during concentric isokinetic muscle actions. J Strength Cond Res. 17: 484-488.

Fowles, J. R., Sale, D. G. and MacDougall, J. D. 2000. Reduced strength after passive stretch of the human plantarflexors. J Appl Physiol (1985). 89: 1179-1188.

Herda, T. J., Cramer, J. T., Ryan, E. D., McHugh, M. P. and Stout, J. R. 2008. Acute effects of static versus dynamic stretching on isometric peak torque, electromyography, and mechanomyography of the biceps femoris muscle. J Strength Cond Res. 22: 809-817. 
Herda, T. J., Ryan, E. D., Costa, P. B., Walter, A. A., Hoge, K. M., Uribe, B. P., McLagan, J. R., Stout, J. R. and Cramer, J. T. 2010. Acute effects of passive stretching and vibration on the electromechanical delay and musculotendinous stiffness of the plantar flexors. Electromyogr Clin Neurophysiol. 50: 277-288.

Herda, T. J., Ryan, E. D., Smith, A. E., Walter, A. A., Bemben, M. G., Stout, J. R. and Cramer, J. T. 2009. Acute effects of passive stretching vs vibration on the neuromuscular function of the plantar flexors. Scand J Med Sci Sports. 19: 703-713.

Kokkonen, J., Nelson, A. G. and Cornwell, A. 1998. Acute muscle stretching inhibits maximal strength performance. Res Q Exerc Sport. 69: 411-415.

Kouzaki, M., Shinohara, M. and Fukunaga, T. 2000. Decrease in maximal voluntary contraction by tonic vibration applied to a single synergist muscle in humans. J Appl Physiol (1985). 89: 1420-1424.

Marek, S. M., Cramer, J. T., Fincher, A. L., Massey, L. L., Dangelmaier, S. M., Purkayastha, S., Fitz, K. A. and Culbertson, J. Y. 2005. Acute Effects of Static and Proprioceptive Neuromuscular Facilitation Stretching on Muscle Strength and Power Output. J Athl Train. 40: 94-103.

McHugh, M. P. and Johnson, A. 2006. Strength Loss Following Static Stretching: The Role of Muscle Length. Medicine and Science in Sports and Exercise. 38: S373-S374.

Muir, I. W., Chesworth, B. M. and Vandervoort, A. A. 1999. Effect of a static calf stretching exercise on the resistive torque during passive ankle dorsiflexion in healthy subjects. Journal of Orthopaedic \& Sports Physical Therapy. 29: 106-113.

Nelson, A. G., Allen, J. D., Cornwell, A. and Kokkonen, J. 2001a. Inhibition of maximal voluntary isometric torque production by acute stretching is joint-angle specific. Res Q Exerc Sport. 72: 68-70.

Nelson, A. G., Guillory, I. K., Cornwell, C. and Kokkonen, J. 2001b. Inhibition of maximal voluntary isokinetic torque production following stretching is velocity-specific. J Strength Cond Res. 15: 241-246.

Nordez, A., Cornu, C. and McNair, P. 2006. Acute effects of static stretching on passive stiffness of the hamstring muscles calculated using different mathematical models. Clinical Biomechanics. 21: 755-760.

Orizio, C., Gobbo, M., Diemont, B., Esposito, F. and Veicsteinas, A. 2003. The surface mechanomyogram as a tool to describe the influence of fatigue on biceps brachii motor unit activation strategy. Historical basis and novel evidence. Eur J Appl Physiol. 90: 326-336. 
Pope, Z. K. and DeFreitas, J. M. 2015. The effects of acute and prolonged muscle vibration on the function of the muscle spindle's reflex arc. Somatosens Mot Res. 32: 254-261.

Richardson, M. S., Cramer, J. T., Bemben, D. A., Shehab, R. L., Glover, J. and Bemben, M. G. 2006. Effects of age and $\mathrm{ACL}$ reconstruction on quadriceps gamma loop function. J Geriatr Phys Ther. 29: 2834.

Ryan, E. D., Beck, T. W., Herda, T. J., Hull, H. R., Hartman, M. J., Stout, J. R. and Cramer, J. T. 2008. Do practical durations of stretching alter muscle strength? A dose-response study. Med Sci Sports Exerc. 40: 1529-1537.

Ryan, E. D., Herda, T. J., Costa, P. B., Herda, A. A. and Cramer, J. T. 2014. Acute effects of passive stretching of the plantarflexor muscles on neuromuscular function: the influence of age. Age (Dordr). 36: 9672.

Ushiyama, J., Masani, K., Kouzaki, M., Kanehisa, H. and Fukunaga, T. 2005. Difference in aftereffects following prolonged Achilles tendon vibration on muscle activity during maximal voluntary contraction among plantar flexor synergists. J Appl Physiol (1985). 98: 1427-1433.

Winter, D. 1990. Biomechanics and motor control of human movement. New York, NY: John Wiley \& Sons Inc.

Young, W. and Elliott, S. 2001. Acute effects of static stretching, proprioceptive neuromuscular facilitation stretching, and maximum voluntary contractions on explosive force production and jumping performance. Res Q Exerc Sport. 72: 273-279. 
Table 1. Mean \pm SD for pre- and post-passive stretching only $(\mathrm{PS}+\mathrm{CON})$ and passive stretching + vibration (PS+VIB) treatments for voluntary peak torque $(\mathrm{PT})$, percent voluntary inactivation $(\% \mathrm{VI})$, resting single twitch torque $\left(\mathrm{TT}_{\mathrm{SINGLE}}\right)$, and resting doublet twitch torque $\left(\mathrm{TT}_{\text {DOUBLET }}\right)$ at the dorsiflexion (DF) and plantarflexion (PF) joint angles.

\begin{tabular}{|c|c|c|c|c|c|c|c|c|}
\hline & \multicolumn{4}{|c|}{$\mathrm{PS}+\mathrm{CON}$} & \multicolumn{4}{|c|}{ PS+VIB } \\
\hline & \multicolumn{2}{|c|}{ DF } & \multicolumn{2}{|c|}{$\mathrm{PF}$} & \multicolumn{2}{|c|}{$\mathrm{DF}$} & \multicolumn{2}{|c|}{$\mathrm{PF}$} \\
\hline & Pre & Post & Pre & Post & Pre & Post & Pre & Post \\
\hline PT (Nm) & $129.1 \pm 35.3$ & $119.1 \pm 34.7$ & $92.3 \pm 21.4$ & $84.0 \pm 21.4$ & $133.8 \pm 47.4$ & $109.1 \pm 45.2$ & $92.7 \pm 22.2$ & $76.3 \pm 22.4$ \\
\hline$\% \mathrm{VI}$ & $24.6 \pm 14.9$ & $27.9 \pm 18.7$ & $7.4 \pm 13.6$ & $18.0 \pm 20.5$ & $20.4 \pm 16.3$ & $34.5 \pm 19.5$ & $8.5 \pm 15.0$ & $21.7 \pm 30.7$ \\
\hline $\mathrm{TT}_{\text {SINGLE }}(\mathrm{Nm})$ & $25.3 \pm 7.8$ & $23.7 \pm 7.8$ & $8.9 \pm 4.6$ & $7.5 \pm 4.2$ & $22.9 \pm 9.2$ & $21.2 \pm 8.1$ & $9.3 \pm 3.9$ & $6.7 \pm 2.4$ \\
\hline $\mathrm{TT}_{\text {DOUBLET }}(\mathrm{Nm})$ & $44.0 \pm 14.5$ & $42.3 \pm 14.9$ & $23.4 \pm 9.4$ & $20.8 \pm 8.8$ & $41.9 \pm 15.3$ & $37.6 \pm 16.2$ & $22.9 \pm 8.6$ & $20.6 \pm 7.6$ \\
\hline
\end{tabular}

Please refer to the results section for significant interactions and main effects. 
Table 2. Mean \pm SD for pre- and post-passive stretching only (PS+CON) and passive stretching + vibration (PS+VIB) treatments for passive range of motion (PROM), muscle tendon stiffness (MTS), and passive torque (PASSTQ).

\begin{tabular}{cccccc} 
& \multicolumn{2}{c}{ PS+CON } & & \multicolumn{2}{c}{ PS+VIB } \\
\cline { 2 - 3 } \cline { 5 - 6 } PROM $\left({ }^{\circ}\right)$ & $30.9 \pm 11.6$ & $34.6 \pm 12.4^{*}$ & & $32.5 \pm 10.4$ & $35.0 \pm 12.8^{*}$ \\
MTS $\left(\mathrm{Nm} /{ }^{\circ}\right)$ & $1.4 \pm 0.9$ & $1.1 \pm 0.6^{*}$ & & $1.422 \pm 1.0$ & $1.1 \pm 0.7^{*}$ \\
PASSTQ $(\mathrm{Nm})$ & $13.1 \pm 4.7$ & $11.2 \pm 3.8^{*}$ & & $13.4 \pm 4.8$ & $11.5 \pm 3.8^{*}$ \\
\hline
\end{tabular}

*Indicates significant difference from pre- to post-passive stretching collapsed across PS+CON and PS+VIB. 


\section{Figure Legend}

Figure 1. Timeline for experimental trials. Post-passive stretching included either no vibration $(\mathrm{PS}+\mathrm{CON})$ or vibration $(\mathrm{PS}+\mathrm{VIB})$ throughout the remainder of testing. $\mathrm{DF}=$ dorsiflexion; $\mathrm{PF}=$ plantarflexion; MVC ITT = maximal voluntary contraction with the interpolated twitch technique; $\mathrm{PROM}=$ passive range of motion; $\mathrm{VIB}=$ vibration.

Figure 2. Percent (\%) change for each subject from pre- to post-passive stretching for the passive stretching $(\mathrm{PS}+\mathrm{CON})$ and passive stretching plus vibration $(\mathrm{PS}+\mathrm{VIB})$ and at both joint angles, $+15^{\circ}$ dorsiflexion (DF), and $-20^{\circ}$ plantarflexion (PF) groups for (A) voluntary peak torque (PT), (B) potentiated twitch torque from doublet stimuli (TT DOUBLET $_{\text {), }}(\mathrm{C})$ resting twitch torque from single stimulus (TT $\left.\mathrm{TINGLE}_{\mathrm{S}}\right)$, and (D) percent voluntary inactivation (\%VI). Circles represent individual changes. Positive values indicate increases and negative values indicate decreases from pre- to post-passive stretching. The larger horizontal bars represent the mean percent change scores and the smaller bars represent $95 \%$ confidence intervals about the means. 


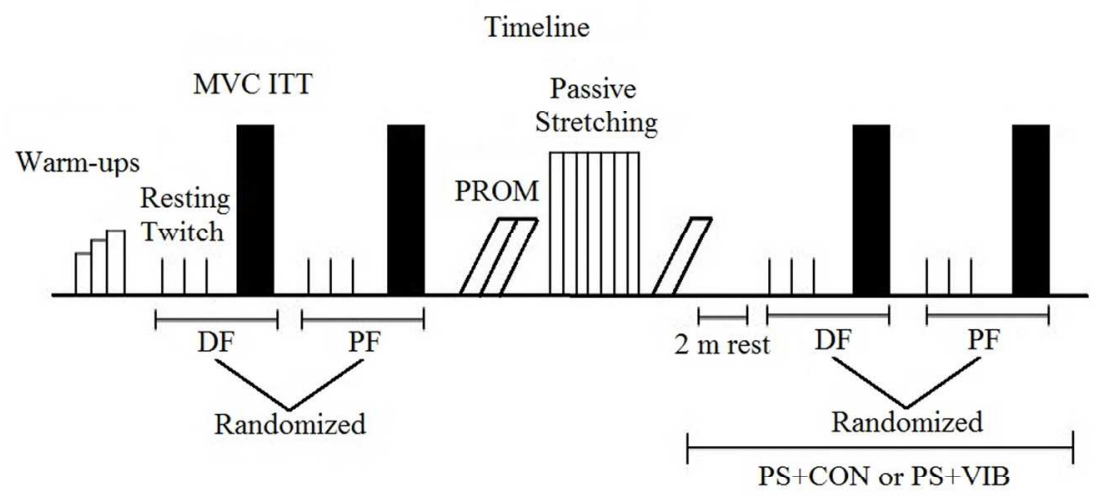

Figure 1. Timeline for experimental trials. Post-passive stretching included either no vibration $(P S+C O N)$ or vibration (PS+VIB) throughout the remainder of testing. DF = dorsiflexion; $\mathrm{PF}=$ plantarflexion; $\mathrm{MVC}$ ITT = maximal voluntary contraction with the interpolated twitch technique; PROM = passive range of motion; VIB $=$ vibration.

$338 \times 190 \mathrm{~mm}(300 \times 300$ DPI $)$ 


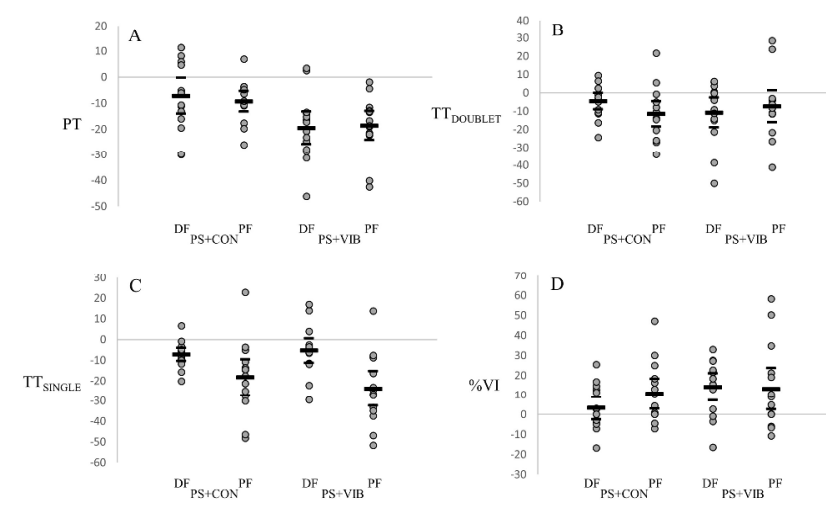

Figure 2. Percent (\%) change for each subject from pre- to post-passive stretching for the passive stretching (PS+CON) and passive stretching plus vibration (PS+VIB) and at both joint angles, $+15^{\circ}$ dorsiflexion (DF), and $-20^{\circ}$ plantarflexion (PF) groups for (A) voluntary peak torque (PT), (B) potentiated twitch torque from doublet stimuli (TTDOUBLET), (C) resting twitch torque from single stimulus (TTSINGLE), and (D) percent voluntary inactivation (\%VI). Circles represent individual changes. Positive values indicate increases and negative values indicate decreases from pre- to post-passive stretching. The larger horizontal bars represent the mean percent change scores and the smaller bars represent $95 \%$ confidence intervals about the means.

$338 \times 190 \mathrm{~mm}(300 \times 300 \mathrm{DPI})$ 